\title{
A INFLUÊNCIA DAS CAPACIDADES DE DETECÇÃO, APREENSÃO E RECONFIGURAÇÃO NO DESENVOLVIMENTO DE CAPACIDADES DINÂMICAS ORIENTADAS À SUSTENTABILIDADE
}

http://dx.doi.org/10.21527/2237-6453.2021.55.11550

Recebido em: 30/9/2020

Aceito em: 12/4/2021

\author{
Felipe Cavalheiro Zaluski, ${ }^{1}$ Clarice Vepo do Nascimento Welter, ${ }^{1}$ Jorge Oneide Sausen ${ }^{2}$, \\ Clandia Maffini Gomes, ${ }^{1}$ Roberto Schoproni Bichueti ${ }^{1}$
}

\begin{abstract}
RESUMO
O estudo buscou analisar a influência das capacidades de detectar oportunidades e ameaças (sensing), capacidade de apreensão de oportunidades (seizing) e capacidade de gerenciar ameaças e transformações (reconfiguring) no desenvolvimento de Capacidades Dinâmicas Sustentáveis em indústrias da região metropolitana de Porto Alegre/ RS. Com natureza descritiva, foi conduzido por meio de uma survey com 77 indústrias de grande porte, com análise quantitativa dos dados por meio da modelagem de equações estruturais. Pode-se concluir que a articulação das capacidades dinâmicas, alinhadas com os conceitos de sustentabilidade, pode auxiliar as empresas no desenvolvimento de capacidades dinâmicas sustentáveis. Quanto às implicações teóricas, o artigo contribui para o avanço dos estudos relacionados às capacidades dinâmicas e sustentabilidade, demonstrando como as organizações, a partir da combinação de seus recursos e rotinas, podem vir a fomentar o desenvolvimento de suas capacidades dinâmicas orientadas à sustentabilidade. Argumenta-se que se as empresas desenvolverem a capacidade de detectar oportunidades e ameaças, apreensão de oportunidades e gerenciamento de ameaças e transformações orientadas à sustentabilidade, migrarão para estratégias e operações que se traduzirão em viabilidade não só econômica, mas também ambiental e social. Desenvolver capacidades dinâmicas orientadas à sustentabilidade, portanto, é um caminho para a sustentabilidade empresarial.
\end{abstract}

Palavras-chave: Capacidades dinâmicas. Sustentabilidade. Rotinas. Processos. Recursos.

\section{THE INFLUENCE OF DETECTION, SEIZING AND RECONFIGURATION CAPACITIES ON THE DEVELOPMENT OF ORIENTED ON SUSTAINABILITY}

\begin{abstract}
The study sought to analyze the influence of the abilities to detect opportunities and threats (sensing), the ability to seize opportunities (seizing) and the ability to manage threats and transformations (reconfiguring) in the development of Sustainable Dynamic Capabilities in industries in the metropolitan region of Porto Alegre/RS. Descriptive in nature, it was conducted through a survey of seventy-seven large industries, with quantitative analysis of the data through the modeling of structural equations. It can be concluded that the articulation of dynamic capabilities in line with sustainability concepts can assist companies in the development of sustainable dynamic capabilities. With relation to the theoretical implications, the article contributes to the advancement of studies related to dynamic capabilities and sustainability, demonstrating how organizations from the combination of their resources and routines can come to foster the development of their dynamic capabilities oriented to sustainability. It is argued that if companies develop the ability to detect opportunities and threats, seize opportunities and manage threats and transformations oriented to sustainability, they will migrate to strategies and operations that will translate into not only economic, but also environmental and social viability. Therefore, developing dynamic capabilities oriented towards sustainability is a path to corporate sustainability.
\end{abstract}

Keywords: Dynamic Capabilities. Sustainability. Routines. Processes. Resources.

\footnotetext{
${ }^{1}$ Autor correspondente. Universidade Federal de Santa Maria (UFSM). Av. Roraima, no 1000 - Cidade Universitária - Bairro Camobi. Santa Maria/RS, Brasil. CEP 97105-900. http://lattes.cnpq.br/5563360256401044. http://orcid.org/0000-0003-0942-9180. felipezaluski@hotmail.com

${ }^{2}$ Universidade Regional do Noroeste do Estado do Rio Grande do Sul (Unijuí). Ijuí/RS, Brasil.
} 


\section{INTRODUÇÃO}

Diante dos crescentes desafios e pressões de governos, organizações não governamentais (ONGs), investidores e funcionários para que as empresas se tornem mais conscientes sobre os impactos ambientais e sociais de suas atividades comerciais, as empresas estão procurando maneiras diferentes de fazer as coisas e também buscando oportunidades de crescimento alinhadas com os aspectos sustentáveis (GERADTS; BOCKEN, 2018).

Neste contexto, a ideia da inserção da sustentabilidade nas estratégias organizacionais tem ganhado importância no contexto empresarial. Ao longo do tempo, as empresas vêm demonstrando capacidade de desenvolvimento de habilidades e competências que as possibilitam enfrentar os desafios de seu ambiente competitivo, mostrando que não somente se adaptam a tal ambiente, mas que também os moldam mediante a inovação e a colaboração com outras empresas, entidades e instituições (TEECE, 2007).

O estudo destas capacidades, reconhecidas na literatura como Capacidades Dinâmicas (CDs), podem ajudar a compreender, por exemplo, o porquê de determinadas empresas conseguirem atingir um patamar de sustentabilidade e manter-se ao longo do tempo, enquanto outras parecem ter maior dificuldade para lidar com este desafio (NAGATA, 2020). As CDs podem ser definidas como a habilidade de integrar, construir, combinar, proteger e reconfigurar recursos, capacidades e ativos tangíveis e intangíveis da organização em resposta às mudanças ambientais (TEECE, 2007; TEECE; PISANO; SHUEN, 1997).

Conforme Wu et al. (2012), a questão de como as empresas desenvolvem e aplicam as CDs para enfrentar os desafios distintos envolvidos nas mudanças corporativas em direção à sustentabilidade, ainda não foi totalmente explorada. De acordo com Amui et al. (2017), a maior parte da literatura sobre a relação entre sustentabilidade e CDs compreende estudos teóricos, carecendo de pesquisas empíricas sobre o assunto. Estudos anteriores fundamentaram e ilustraram como ocorre o processo de desenvolvimento das CDs (TONDOLO; BITENCOURT, 2014; ZALUSKI; SAUSEN; FERREIRA, 2020), no entanto poucos estudos exploram esse processo em prol do desenvolvimento de capacidades dinâmicas sustentáveis (MOUSAVI; BOSSINK; VAN VLIET, 2018).

Diante deste contexto, propõem-se a seguinte questão: As capacidades de deteç̧ão, apreensão e reconfiguração influenciam positivamente no desenvolvimento de capacidades dinâmicas orientadas à sustentabilidade? Para compreender estas relações, o objetivo deste estudo é analisar a influência das capacidades de detectar oportunidades e ameaças, apreensão de oportunidades e gerenciamento de ameaças e transformações no desenvolvimento de Capacidades Dinâmicas Sustentáveis em indústrias da região metropolitana de Porto Alegre/RS. Para isso, adaptou-se os modelos de Teece (2007) e Nagata (2020) em dimensões e indicadores que resultam em um modelo teórico para análise de habilidades, competências e rotinas organizacionais das capacidades de detecção, apreensão e reconfiguração, que possibilitam compreender o desenvolvimento de CDs orientadas à sustentabilidade em organizações. De natureza descritiva, este estudo foi conduzido por intermédio de uma survey, com análise quantitativa dos dados por meio da Modelagem de Equações Estruturais - Partial Least Squares (MEE-PLS).

Optou-se pela realização do estudo em indústrias de grande porte da região metropolitana de Porto Alegre/RS pela representatividade da indústria na economia brasileira e, conse- 
A INFLUÊNCIA DAS CAPACIDADES DE DETECÇÃO, APREENSÃO E RECONFIGURAÇÃO NO DESENVOLVIMENTO DE CAPACIDADES DINÂMICAS ORIENTADAS À SUSTENTABILIDADE

Felipe Cavalheiro Zaluski - Clarice Vepo do Nascimento Welter - Jorge Oneide Sausen Clandia Maffini Gomes - Roberto Schoproni Bichueti

quentemente, no Rio Grande do Sul. Segundo dados do IBGE no ano de 2019, a atividade desta região se saiu melhor que a média do Estado. Soma-se a isso a crescente preocupação destas indústrias com relação à sustentabilidade, pois, de acordo com a Federação das Indústrias do Estado do Rio Grande do Sul (FIERGS, 2019), a sociedade gaúcha tem pressa quanto à aprovação de reformas que recoloquem o Rio Grande do Sul no caminho do desenvolvimento sustentável.

A pesquisa aponta influências positivas entre as capacidades de detectar oportunidades e ameaças (sensing), capacidade de apreensão de oportunidades (seizing) e capacidade de gerenciar ameaças e transformações (reconfiguring) no desenvolvimento de CDs orientadas à sustentabilidade. Os resultados contribuem com os estudos no campo das CDs ao trazer informações de como as organizações, a partir de tais capacidades, podem vir a combinar recursos, rotinas e processos da organização para alavancar sustentabilidade.

\section{REFERENCIAL TEÓRICO}

Na sequência apresenta-se, primeiramente, os conceitos e evolução da temática da sustentabilidade. Após, destaca-se a temática das capacidades dinâmicas bem como as hipóteses que norteiam este estudo.

\section{Sustentabilidade}

Em decorrência dos grandes avanços tecnológicos da atualidade, o mundo dos negócios tem passado por uma transformação gigantesca diante do aumento da preocupação com as questões ambientais e sociais por parte de clientes, investidores e sociedade, que passaram a exigir das empresas uma postura mais consciente acerca dos impactos ambientais e sociais das suas atividades (GERADTS; BOCKEN, 2018; LOREDO et al., 2019; GLOBOCNIK; RAUTER; BAUMGARTNER, 2020).

O modelo de consumo e produção vigente, que tem como principal objetivo a lucratividade a qualquer preço, fez com que a exploração de recursos se intensificasse, causando grandes impactos, que afetam drasticamente os diversos compartimentos ambientais (ABRAMOVAY, 2012). O grande desafio para as organizações contemporâneas consiste em encontrar soluções para ajustar o crescimento econômico e o cuidado com as questões ambientais e sociais (FROEHLICH; BITENCOURT, 2015; RENZI; HENZ; RIPPEL, 2019).

Sob essa linha de ação, só é sustentável se for possível manter, ao invés de esgotar, as fontes de riqueza (capital artificial, social, cultural, humana, financeira e natural), a fim de deixar para as gerações futuras um estoque de capital capaz de proporcionar o mesmo bem-estar atual. Essa mudança necessária não abrange somente modelos de negócios, mas, também, padrões econômicos e de consumo mais amplos para que se consiga a sustentabilidade tanto econômica, quanto ambiental e social (AVESANI, 2020).

Neste sentido, executivos e estudiosos passaram a destacar fortemente que as organizações devem responder adequadamente às questões econômicas e às necessidades dos clientes, ao mesmo tempo em que consideram o bem-estar humano e as restrições ecológicas (GELHARD; VON DELFT, 2016). Percebe-se, também, um número crescente de estudos que mostra que a mudança sustentável das estratégias e operações estabelecidas de uma empresa acabará 
A INFLUÊNCIA DAS CAPACIDADES DE DETECÇÃO, APREENSÃO E RECONFIGURAÇÃO NO DESENVOLVIMENTO DE CAPACIDADES DINÂMICAS ORIENTADAS À SUSTENTABILIDADE

Felipe Cavalheiro Zaluski - Clarice Vepo do Nascimento Welter - Jorge Oneide Sausen Clandia Maffini Gomes - Roberto Schoproni Bichueti

por se traduzir em sua viabilidade econômica e vantagem competitiva sustentada (HART, 1995; RUSSO; FOUTS, 1997; PORTER; KRAMER, 2006; HART; DOWELL, 2011).

A noção mais ampla de sustentabilidade pode ser entendida como a manutenção do equilíbrio ao longo do tempo dos recursos ambientais, que seja capaz de suprir as necessidades humanas das gerações presentes e futuras, a qual está alinhada ao conceito de desenvolvimento sustentável difundido pela Comissão de Brundtland (CMMAD, 1991). De acordo com o documento, todo desenvolvimento deveria orientar-se pela noção de que os recursos naturais são uma herança a ser deixada para as gerações futuras e que, por isso, devemos protegê-los e usá-los com consciência.

Na visão de Nagata (2020), a sustentabilidade refere-se à capacidade de manter algo em um estado contínuo. Quando considerada no contexto organizacional, a sustentabilidade refere-se à capacidade de manter a empresa em estado de equilíbrio do ponto de vista ambiental, econômico e social ao longo do tempo. Essas dimensões formam o Triple Bottom Line (TBL), termo cunhado na década de 90 do século 20 por John Eklington, que sugeriu um modelo que traduzisse a ideia de desenvolvimento sustentável para a linguagem empresarial (LEDERWASCH; MUKHEIBIR, 2013; ALHADDI, 2015). Esse conceito sugere que a sustentabilidade está associada à obtenção, por parte das empresas, de resultados positivos não somente em termos econômicos, mas também nos aspectos sociais e ambientais (NAGATA, 2020). A sustentabilidade é, portanto, um convite a uma abordagem interdisciplinar ao lidar com questões de desenvolvimento.

Manter o equilíbrio entre as três dimensões, porém, tem sido o grande desafio para as organizações contemporâneas, que necessitam se adaptar às constantes pressões do ambiente, de modo a inserir a sustentabilidade em seus modelos de negócios, mudando para modos de produção e consumo mais sustentáveis. A busca simultânea de objetivos econômicos, ambientais e sociais está rapidamente tornando-se uma prioridade estratégica para empresas de diferentes setores (WU et al., 2012). Embora ela tenha sido apresentada de forma desmembrada, as três sustentabilidades (ambiental, econômica e social) devem acontecer de forma articulada para a realização tanto da sustentabilidade na organização quanto para um desenvolvimento sustentável mais amplo (HANSMANN; MIEG; FRISCHKNECHT, 2012).

Embora, todavia, a sustentabilidade tenha passado a ser vista como fonte de vantagem competitiva, Wu et al. (2012) argumentam que nem todas as empresas seguirão o mesmo caminho rumo à sustentabilidade; em parte em razão de restrições de investimento e da troca entre retornos dos modelos de negócios tradicionais e o custo de mudanças sustentáveis, e em parte pelas diversas capacidades necessárias para lidar com o relacionamento entre os negócios sociais e ambientais.

Que capacidades, entretanto, são necessárias para que as organizações consigam se tornar mais sustentáveis? Nagata (2020) identificou uma série de variáveis estudadas ligadas a CDs que favorecem a sustentabilidade e sua aderência ao modelo de Teece (2007). De posse destas variáveis, torna-se possível a verificação de como estas CDs, voltadas para a Sustentabilidade, são desenvolvidas pelas organizações e os padrões existentes neste processo de desenvolvimento destas CDs orientadas à sustentabilidade. Deste modo, faz-se necessário uma compreensão mais aprofundada sobre Capacidades Dinâmicas, o que será feito na próxima seção. 


\section{Capacidades Dinâmicas}

A proposta original acerca das Capacidades Dinâmicas (CDs) foi de Winter (1964). O tema, no entanto, passou a ser desenvolvido mais enfaticamente a partir da segunda metade da década de 90 do século 20, constituindo-se em um ramo de grande interesse para pesquisadores na área de administração em campos diversos do conhecimento, como gerenciamento estratégico, empreendedorismo, marketing, gestão de recursos humanos, gestão de operações, inclusive sistema de informação (MEIRELLES; CAMARGO, 2014; GUERRA; TONDOLO; CAMARGO, 2016).

Teece, Pisano e Shuen (1997) aprimoraram o conceito e definiram as CDs como "a capacidade da empresa em integrar, criar e reconfigurar interna e externamente competências ao responder a ambientes em rápida mudança" (p. 516, tradução nossa). Neste sentido, a perspectiva das CDs surge como um apoio às empresas que procuram estabelecer uma vantagem competitiva em mercados dinâmicos e com elevados níveis de exigência.

Refinando o estudo supracitados, Eisenhardt e Martin (2000) conceituaram CDs como processos empresariais que utilizam recursos, definidos como "as rotinas organizacionais e estratégicas pelas quais as empresas alcançam novas configurações de recursos, como mercados que emergem, colidem, dividem, evoluem e morrem" (p. 1.107, tradução nossa). Esta definição enfatiza mais a ideia de rotina e de recurso em detrimento de habilidade ou competência, utilizado por Teece, Pisano e Shuen (1997).

Dado o escopo e a complexidade do assunto, diferentes estudos concentraram-se em elementos específicos de CDs. Meirelles e Camargo (2014) afirmam que, em linhas gerais, é possível identificar, nas várias definições de CDs, duas linhas de abordagem: i) conjunto de habilidades, comportamentos e capacidades organizacionais; ii) conjunto de rotinas e processos. Esses autores apresentam os estudiosos que tratam as CDs como conjunto de capacidades e habilidades, em que se destacam as visões de Collis (1994), Andreeva e Chaika (2006) e McKelvie e Davidson (2009). No grupo de autores que tratam as CDs como rotinas e processos, destacam-se as visões de Eisenhardt e Martin (2000), Winter (2003), Zollo e Winter (2002), Bygdas (2006), Dosi, Faillo e Marengo (2008), Teece, Pisano, Shuen (1997) e Teece $(2007,2009)$. A Tabela 1 resume os conceitos existentes na literatura sobre CDs de acordo com essas duas abordagens.

Percebe-se que são várias as definições, algumas muito semelhantes e, principalmente, nota-se fortes controvérsias sobre os condicionantes (antecendentes) e elementos componentes das CDS (MEIRELLES; CAMARGO, 2014). Diante dos conceitos até aqui apresentados, constata-se que capacidade dinâmica é a habilidade das organizações de identificar, instituir, reconfigurar e renovar a sua base de recursos, tanto tangíveis quanto intangíveis, bem como as suas competências, sendo um conceito de gerenciamento estratégico usado para obter vários benefícios e vantagens para as empresas, incluindo o desenvolvimento de vantagem competitiva (SUNDER; GANESH; MARATHE, 2019).

Teece (2007) dividiu as CDs em três capacidades ou dimensões: a) perceber e moldar oportunidades e ameaças (Sense); b) aproveitar oportunidades (Seize); e c) gerenciar ameaças e reconfiguração (Reconfiguring). $O$ autor também propôs uma série de indicadores ou microfundamentos originados na literatura sobre estratégia, inovação e organização (TEECE, 2007). A Figura 1 ilustra os resultados da integração dessas diferentes perspectivas. 
A INFLUÊNCIA DAS CAPACIDADES DE DETECÇÃO, APREENSÃO E RECONFIGURAÇÃO NO DESENVOLVIMENTO DE CAPACIDADES DINÂMICAS ORIENTADAS À SUSTENTABILIDADE

Felipe Cavalheiro Zaluski - Clarice Vepo do Nascimento Welter - Jorge Oneide Sausen Clandia Maffini Gomes - Roberto Schoproni Bichueti

Tabela 1 - Capacidades dinâmicas como conjunto de habilidades, comportamentos e capacidades organizacionais e como rotinas e processos

\begin{tabular}{|c|c|c|}
\hline & AUTORES & DEFINIÇÃO \\
\hline \multirow{7}{*}{$\begin{array}{l}\text { Definição de } \\
\text { capacidade } \\
\text { dinâmica como } \\
\text { conjunto de } \\
\text { habilidades, } \\
\text { comportamentos } \\
\text { e capacidades } \\
\text { organizacionais }\end{array}$} & Collis (1994) & $\begin{array}{l}\text { Capacidade em inovar mais rapidamente ou de forma melhor } \\
\text { do que a concorrência. }\end{array}$ \\
\hline & $\begin{array}{l}\text { Lee, Lee e Rho } \\
\text { (2002) }\end{array}$ & $\begin{array}{l}\text { Uma fonte mais recente de vantagem competitiva no que se } \\
\text { refere à forma de a empresa enfrentar ou até mesmo criar uma } \\
\text { mudança de mercado. }\end{array}$ \\
\hline & $\begin{array}{l}\text { Zahra e George } \\
\qquad(2002)\end{array}$ & $\begin{array}{l}\text { São capacitações direcionadas à mudança, que ajudam a } \\
\text { empresa a reconfigurar os seus recursos de forma a enfrentar a } \\
\text { crescente exigência do mercado e as estratégias da concorrência }\end{array}$ \\
\hline & $\begin{array}{l}\text { Andreeva e } \\
\text { Chaika (2006) }\end{array}$ & $\begin{array}{l}\text { Capacidades dinâmicas são aquelas que habilitam a organização } \\
\text { a renovar suas competências-chave conforme ocorrem } \\
\text { mudanças no ambiente operacional. }\end{array}$ \\
\hline & $\begin{array}{l}\text { Helfat et al. } \\
\qquad(2007)\end{array}$ & $\begin{array}{l}\text { Capacidade de uma organização de criar, estender ou modificar } \\
\text { sua base de recursos propositadamente. }\end{array}$ \\
\hline & $\begin{array}{l}\text { Wang e Ahmed } \\
\qquad(2007)\end{array}$ & $\begin{array}{l}\text { Comportamento constantemente orientado a integrar, } \\
\text { reconfigurar, renovar e recriar seus recursos e capacidades, e } \\
\text { melhorar e reconstruir as capacidades-chave em resposta às } \\
\text { mutações do ambiente para atingir e sustentar a vantagem } \\
\text { competitiva. }\end{array}$ \\
\hline & $\begin{array}{c}\text { McKelvie e } \\
\text { Davidson (2009) }\end{array}$ & Capacidades dinâmicas como um feixe de outras capacidades. \\
\hline \multirow{9}{*}{$\begin{array}{l}\text { Definição de } \\
\text { capacidade } \\
\text { dinâmica como } \\
\text { conjunto, rotinas e } \\
\text { processos }\end{array}$} & $\begin{array}{l}\text { Eisenhardt e } \\
\text { Martin (2000) }\end{array}$ & $\begin{array}{l}\text { CDs são rotinas organizacionais e estratégicas que possibilitam } \\
\text { novas configurações de recursos em mercados em mudança. }\end{array}$ \\
\hline & $\begin{array}{l}\text { Zollo e Winter } \\
\qquad(2002)\end{array}$ & $\begin{array}{l}\text { Padrão aprendido e estável de atividade coletiva por meio } \\
\text { do qual a organização sistematicamente gera e modifica suas } \\
\text { rotinas operacionais em busca de melhoria na efetividade. }\end{array}$ \\
\hline & Winter (2003) & $\begin{array}{l}\text { Capacidades para operar, estender, modificar ou criar } \\
\text { capacidades comuns. }\end{array}$ \\
\hline & Bygd & $\begin{array}{l}\text { Processos de ativar estruturas distribuídas de conhecimento } \\
\text { e redes fragmentadas de procedimentos e entendimentos } \\
\text { soltos que desenvolvem práticas mais eficientes que não são } \\
\text { facilmente imitáveis. }\end{array}$ \\
\hline & $\begin{array}{l}\text { Dosi, Faillo e } \\
\text { Marengo (2008) }\end{array}$ & $\begin{array}{l}\text { Heurísticas gerenciais e as ferramentas de diagnósticos } \\
\text { constituem o cerne das capacidades dinâmicas. }\end{array}$ \\
\hline & $\begin{array}{l}\text { Teece, Pisano e } \\
\text { Shuen (1997) }\end{array}$ & $\begin{array}{l}\text { Habilidade da firma em integrar, construir e reconfigurar } \\
\text { competências, interna e externamente, para endereçar } \\
\text { ambientes em rápida mudança. }\end{array}$ \\
\hline & $\begin{array}{l}\text { Teece }(2007, \\
\text { 2009) }\end{array}$ & $\begin{array}{l}\text { Capacidade de sentir o contexto do ambiente; aproveitar } \\
\text { oportunidades; gerenciar ameaças e transformações. }\end{array}$ \\
\hline & $\begin{array}{l}\text { Protogerou, } \\
\text { Caloghirou e } \\
\text { Lioukas (2011) }\end{array}$ & $\begin{array}{l}\text { Capacidades dinâmicas são definidas como processos de ordem } \\
\text { maior. que integram, recombinam e geram novas capacidades } \\
\text { tecnológicas e de mercado que, por sua vez, afetam o } \\
\text { desempenho da empresa. }\end{array}$ \\
\hline & $\begin{array}{l}\text { Cordes-Berszinn } \\
\qquad(2013)\end{array}$ & $\begin{array}{l}\text { Capacidades dinâmicas são processos gerenciais e } \\
\text { organizacionais que replicam e reconfiguram recursos } \\
\text { organizacionais dependentes da trajetória. }\end{array}$ \\
\hline
\end{tabular}

Fonte: Elaboração própria. 
Figura 1 - Microfundamentos das CDs

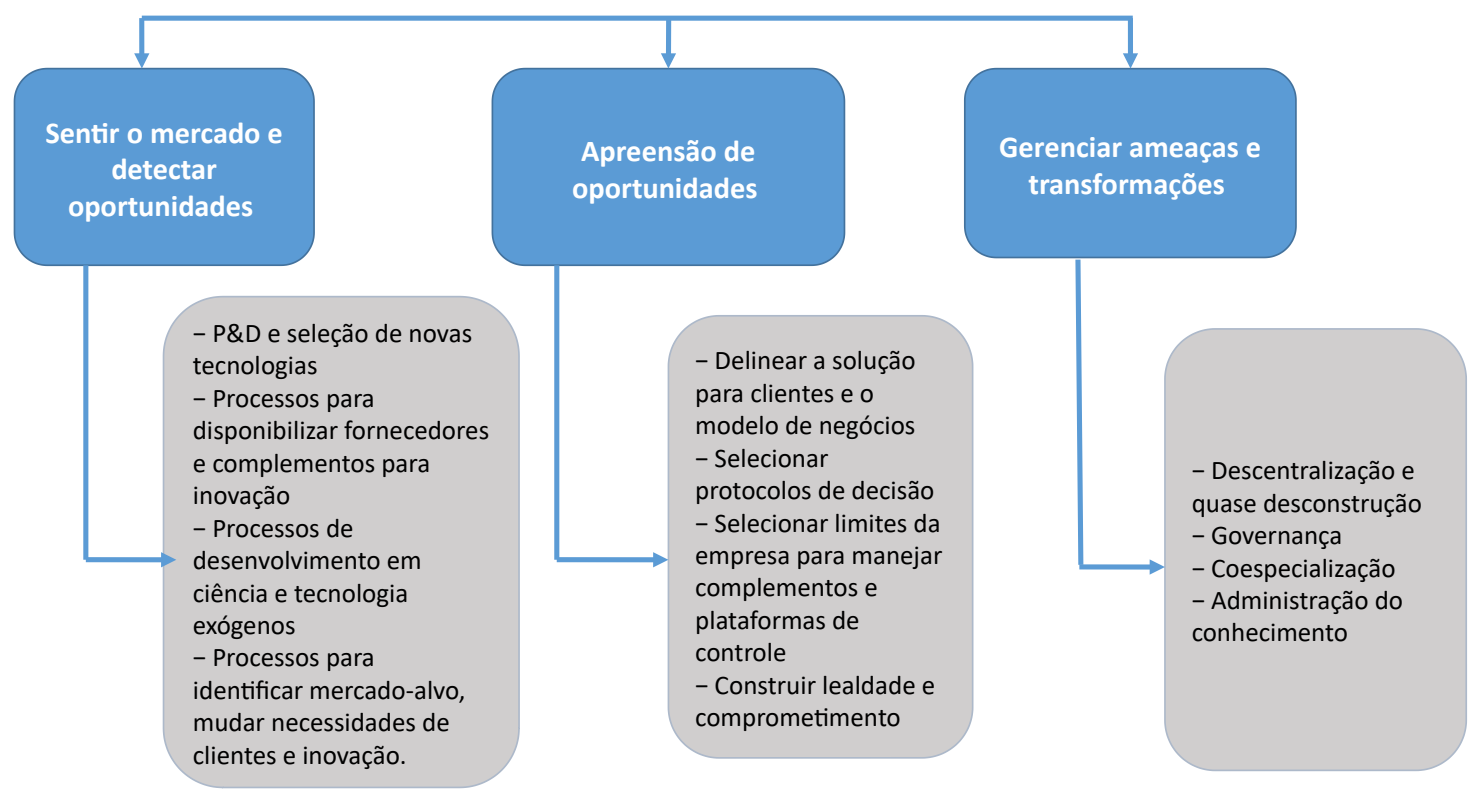

Fonte: Elaborada com base em TEECE (2007).

A capacidade de deteç̧ão de oportunidades e ameaças delimita-se nas atividades de interpretar, aprender, criar e analisar, complementada com investimentos em pesquisa e desenvolvimento (P\&D) (TEECE, 2007). A capacidade de apreensão de oportunidades constitui-se das estruturas empresariais, procedimentos, desenhos e incentivos para aproveitar oportunidades, pois, uma vez identificada, deve ser internalizada para se materializar na oferta de novos produtos, processos ou serviços (NAGATA, 2020).

A capacidade de gerenciar ameaças e transformações é relacionada aos recursos e estruturas das empresas à medida que ela cresce e os mercados e a tecnologia mudam. Teece (2007) menciona, como essencial, potenciar e alcançar a descentralização nas organizações. A flexibilização das estruturas organizacionais com a descentralização das decisões, para os níveis mais baixos da hierarquia, garante uma identificação mais efetiva e no tempo adequado das oportunidades e ameaças.

Neste estudo adaptou-se os modelos de Teece (2007) e Nagata (2020) em dimensões e indicadores que resultam em um modelo teórico para análise de habilidades, competências e rotinas organizacionais das capacidades de detecção, apreensão e reconfiguração que possibilitam compreender o desenvolvimento de capacidades dinâmicas ligadas à sustentabilidade em organizações.

Ponderando que as capacidades de detectar oportunidade de ameaças, apreensão de oportunidades e gerenciamento de ameaças e transformações podem facilitar a mudança estratégica das empresas em relação à sustentabilidade e maior vantagem competitiva em um ambiente de mercado em evolução (WU et al., 2012), têm-se as seguintes hipótese de pesquisa:

$\mathrm{H} 1$ - $\mathrm{O}$ desenvolvimento da capacidade de detectar oportunidades e ameaças (sensing) influencia positivamente o desenvolvimento de capacidades dinâmicas sustentáveis. 
A INFLUÊNCIA DAS CAPACIDADES DE DETECÇÃO, APREENSÃO E RECONFIGURAÇÃO NO DESENVOLVIMENTO DE CAPACIDADES DINÂMICAS ORIENTADAS À SUSTENTABILIDADE

Felipe Cavalheiro Zaluski - Clarice Vepo do Nascimento Welter - Jorge Oneide Sausen Clandia Maffini Gomes - Roberto Schoproni Bichueti

H2 - O desenvolvimento da capacidade de apreensão oportunidades (seizing) influencia positivamente o desenvolvimento de capacidades dinâmicas sustentáveis.

H3 - O desenvolvimento da capacidade de gerenciar ameaças e transformações (reconfiguring) influencia positivamente o desenvolvimento de capacidades dinâmicas sustentáveis.

\section{METODOLOGIA}

A pesquisa adotou uma abordagem quantitativa e descritiva dos dados (RICHARDSON, 2017). Para a escolha do universo amostral delimitou-se a região metropolitana de Porto Alegre, localizada no Estado do Rio Grande do Sul. Deste modo, para eleição das organizações delimitou-se o segmento industrial de grande porte, utilizando a base de dados da Fiergs. Ao todo foram 77 empresas de grande porte associadas à Fiergs.

Optou-se pela seleção de indústrias de grande porte, pois empresas desses portes geralmente possuem uma estrutura interna desenvolvida e formalizada. Os respondentes foram delimitados por cargos de nível estratégico das organizações. Assume-se a limitação pela utilização de um único informante, pois as organizações, em razão de tempo e custos, tendem a não aceitar convites para colaborar com estudos quando são solicitadas respostas de múltiplos informantes (HUBNER; POWER, 1985) e, tendo em vista o objetivo e o contexto de aplicação deste estudo, a escolha de um único informante foi adotada a fim de evitar possíveis perdas de organizações e informações.

A coleta de dados baseou-se em survey, encaminhada via e-mail para as organizações por intermédio de uma plataforma de e-survey. $O$ instrumento de coleta de dados foi elaborado com base na adaptação dos microfundamentos das CDs de Teece (2007) e nos indicadores propostos por Nagata (2020). Uma versão preliminar do instrumento de coleta de dados foi pré-avaliada por três pesquisadores doutores com conhecimento prévios na temática, sendo solicitado que eles avaliassem a relação teórica das variáveis latentes e os indicadores propostos. Após ajustes, uma nova versão foi avaliada por dez respondentes do universo amostral, sendo solicitado que eles fornecessem um feedback sobre a clareza, abrangência, adequação e legibilidade das afirmativas do instrumento.

A Tabela 2 apresenta o modelo teórico das variáveis latentes e indicadores do instrumento de coleta de dados. 
A INFLUÊNCIA DAS CAPACIDADES DE DETECÇÃO, APREENSÃO E RECONFIGURAÇÃO NO DESENVOLVIMENTO

DE CAPACIDADES DINÂMICAS ORIENTADAS À SUSTENTABILIDADE

Felipe Cavalheiro Zaluski - Clarice Vepo do Nascimento Welter - Jorge Oneide Sausen

Clandia Maffini Gomes - Roberto Schoproni Bichueti

Tabela 2 - Modelo teórico das variáveis latentes e indicadores

\begin{tabular}{|c|c|c|c|}
\hline $\begin{array}{l}\text { VL 2a } \\
\text { ORDEM }\end{array}$ & VL 1a ORDEM & INDICADOR & AFIRMATIVA \\
\hline \multirow{15}{*}{$\begin{array}{c}\text { CAPDIN } \\
\text { (Capacidades } \\
\text { dinâmicas } \\
\text { sustentáveis) }\end{array}$} & \multirow{7}{*}{$\begin{array}{c}\text { SENS } \\
\text { (detectar } \\
\text { oportunidades } \\
\text { e ameaças) }\end{array}$} & SENS1 & $\begin{array}{l}\text { A organização adota soluções e tecnologias visando à } \\
\text { sustentabilidade. }\end{array}$ \\
\hline & & SENS2 & $\begin{array}{l}\text { A organização possui programas de desenvolvimento } \\
\text { ou colaboração tecnológica. }\end{array}$ \\
\hline & & SENS3 & $\begin{array}{l}\text { A empresa possui colaboração com os parceiros e } \\
\text { fornecedores para desenvolvimento de soluções. }\end{array}$ \\
\hline & & SENS4 & $\begin{array}{l}\text { A organização possui capacidade de percepção e } \\
\text { iniciativa para mudança. }\end{array}$ \\
\hline & & SENS5 & $\begin{array}{l}\text { A organização possui capacidade de adaptar as } \\
\text { mudanças externas e internas. }\end{array}$ \\
\hline & & SENS6 & $\begin{array}{l}\text { A organização possui capacidade de inovar para instituir } \\
\text { soluções sustentáveis. }\end{array}$ \\
\hline & & SENS7 & A organização analisa e monitora o mercado. \\
\hline & \multirow{5}{*}{$\begin{array}{l}\text { SEIZ } \\
\text { (Apreensão de } \\
\text { oportunidades) }\end{array}$} & SEIZ1 & $\begin{array}{l}\text { A organização estabelece e cumpre padrões/normas } \\
\text { como SGQs e SGAs e obtenção e manutenção de } \\
\text { certificações (como ISO 14001). }\end{array}$ \\
\hline & & SEIZ2 & A organização realiza ações sustentáveis. \\
\hline & & SEIZ3 & $\begin{array}{l}\text { A organização efetua parcerias e relações com outras } \\
\text { organizações. }\end{array}$ \\
\hline & & SEIZ4 & $\begin{array}{l}\text { A empresa cria novos produtos com propostas de valor } \\
\text { sustentável para o mercado. }\end{array}$ \\
\hline & & SEIZ5 & $\begin{array}{l}\text { A organização possui um sistema de gestão ambiental e } \\
\text { visão sustentável do negócio. }\end{array}$ \\
\hline & \multirow{3}{*}{$\begin{array}{l}\text { REC } \\
\text { (Gerenciar } \\
\text { ameaças e }\end{array}$} & REC1 & $\begin{array}{l}\text { A organização possui processos e rotinas para } \\
\text { aprendizagem organizacional. }\end{array}$ \\
\hline & & REC2 & $\begin{array}{l}\text { A organização adota critérios e processos para garantir } \\
\text { a transparência. }\end{array}$ \\
\hline & & REC3 & $\begin{array}{l}\text { A organização possui recursos intangíveis para viabilizar } \\
\text { a estratégia ambiental (como cultura, reputação, capital } \\
\text { intelectual, conhecimento, inovação, qualidade, marca } \\
\text { e finanças...). }\end{array}$ \\
\hline
\end{tabular}

Fonte: Elaborada com base em TEECE (2007); NAGATA (2020).

As variáveis foram medidas por escala tipo Likert de cinco pontos, de "discordo totalmente" (1) a "concordo totalmente" (5) (REVILLA; SARIS; KROSNICK, 2014). A coleta de dados teve retorno de 92 questionários, e 15 apresentaram dados incompletos (missing values). Logo, a amostra final foi de 77 respondentes. A Tabela 3 apresenta a caracterização da amostra respondente e das indústrias. 
A INFLUÊNCIA DAS CAPACIDADES DE DETECÇÃO, APREENSÃO E RECONFIGURAÇÃO NO DESENVOLVIMENTO

DE CAPACIDADES DINÂMICAS ORIENTADAS À SUSTENTABILIDADE

Felipe Cavalheiro Zaluski - Clarice Vepo do Nascimento Welter - Jorge Oneide Sausen

Clandia Maffini Gomes - Roberto Schoproni Bichueti

Tabela 3 - Análise descritiva da amostra de respondentes e das indústrias

\begin{tabular}{|c|c|c|c|c|}
\hline & & Perfil & número & percentual \\
\hline \multirow{13}{*}{$\begin{array}{l}\text { Perfil dos } \\
\text { respondentes }\end{array}$} & \multirow{2}{*}{ Gênero } & Feminino & 51 & $66.2 \%$ \\
\hline & & Masculino & 26 & $33.8 \%$ \\
\hline & \multirow{3}{*}{ Faixa Etária } & de 21 a 30 anos & 8 & $10.4 \%$ \\
\hline & & de 31 a 40 anos & 45 & $58.4 \%$ \\
\hline & & Mais de 40 anos & 24 & $31.2 \%$ \\
\hline & \multirow{3}{*}{ Escolaridade } & Ensino Superior & 12 & $15.6 \%$ \\
\hline & & Pós-Graduação & 56 & $72.7 \%$ \\
\hline & & Mestrado/Doutorado & 9 & $11.7 \%$ \\
\hline & \multirow{5}{*}{$\begin{array}{c}\text { Tempo na } \\
\text { Organização }\end{array}$} & Até 3 anos & 5 & $6.5 \%$ \\
\hline & & De 3 anos até 5 anos & 9 & $11.7 \%$ \\
\hline & & De 5 anos até 8 anos & 22 & $28.6 \%$ \\
\hline & & De 8 anos até 10 anos & 31 & $40.3 \%$ \\
\hline & & Mais de 10 anos & 10 & $13.0 \%$ \\
\hline \multirow{8}{*}{$\begin{array}{l}\text { Perfil das } \\
\text { Indústrias }\end{array}$} & \multirow{8}{*}{ Setor Industrial } & Construção & 21 & $27,3 \%$ \\
\hline & & Alimentos & 18 & $23,4 \%$ \\
\hline & & Automobilística & 2 & $2,6 \%$ \\
\hline & & Máquinas e Equipamentos & 11 & $14,3 \%$ \\
\hline & & Implementos Agrícolas & 7 & $9,1 \%$ \\
\hline & & Vestuário & 6 & $7,8 \%$ \\
\hline & & Metalomecânico & 4 & $5,2 \%$ \\
\hline & & Produtos Químicos & 8 & $10,4 \%$ \\
\hline
\end{tabular}

Fonte: Elaboração própria.

Para testar as hipóteses propostas no estudo, utilizou-se a técnica de mínimos quadrados parciais (PLS), que é um método de modelagem de equações estruturais baseado em variância (SEM). O PLS permite simultaneamente a avaliação do modelo de mensuração, que analisa a confiabilidade e a validade das medidas de construtos teóricos e a avaliação do modelo estrutural que analisa e estima as relações entre esses construtos.

O uso do PLS-SEM é a técnica mais apropriada para este estudo de acordo com os critérios estabelecidos por Roldán e Sánchez-Franco (2012). Quando a amostra ( $n=77)$ é pequena a técnica PLS deve ser aplicada quando o número de observações é menor que 250 casos, ou o objetivo do estudo é a predição das variáveis dependentes, ou, ainda, quando o modelo de pesquisa é consideravelmente complexo de acordo com o tipo de relacionamento nas hipóteses (primeira ordem e segunda ordem). Deste modo, para análise PLS-SEM utilizou-se o software SmartPLS 3.3.2 (RINGLE; SILVA; BIDO, 2014). 


\section{ANÁLISE E DISCUSSÃO DOS RESULTADOS}

Conforme Roldán e Sánchez-Franco (2012), o modelo teórico baseado no PLS-SEM é analisado e interpretado em duas fases. A primeira avalia a confiabilidade e a validade convergente e discriminante do modelo de mensuração, e a segunda fase busca testar as hipóteses do modelo estrutural. Essa sequência de avaliações garante que as medidas dos construtos sejam válidas e confiáveis antes de se tentar tirar conclusões sobre as relações entre os construtos. Após as análises PLS-SEM, efetua-se uma discussão teórica quanto às hipóteses analisadas. A Figura 2 apresenta o modelo estrutural desenvolvido para as análises.

Figura 2 - Especificação do modelo estrutural no SmartPLS 3.3.2

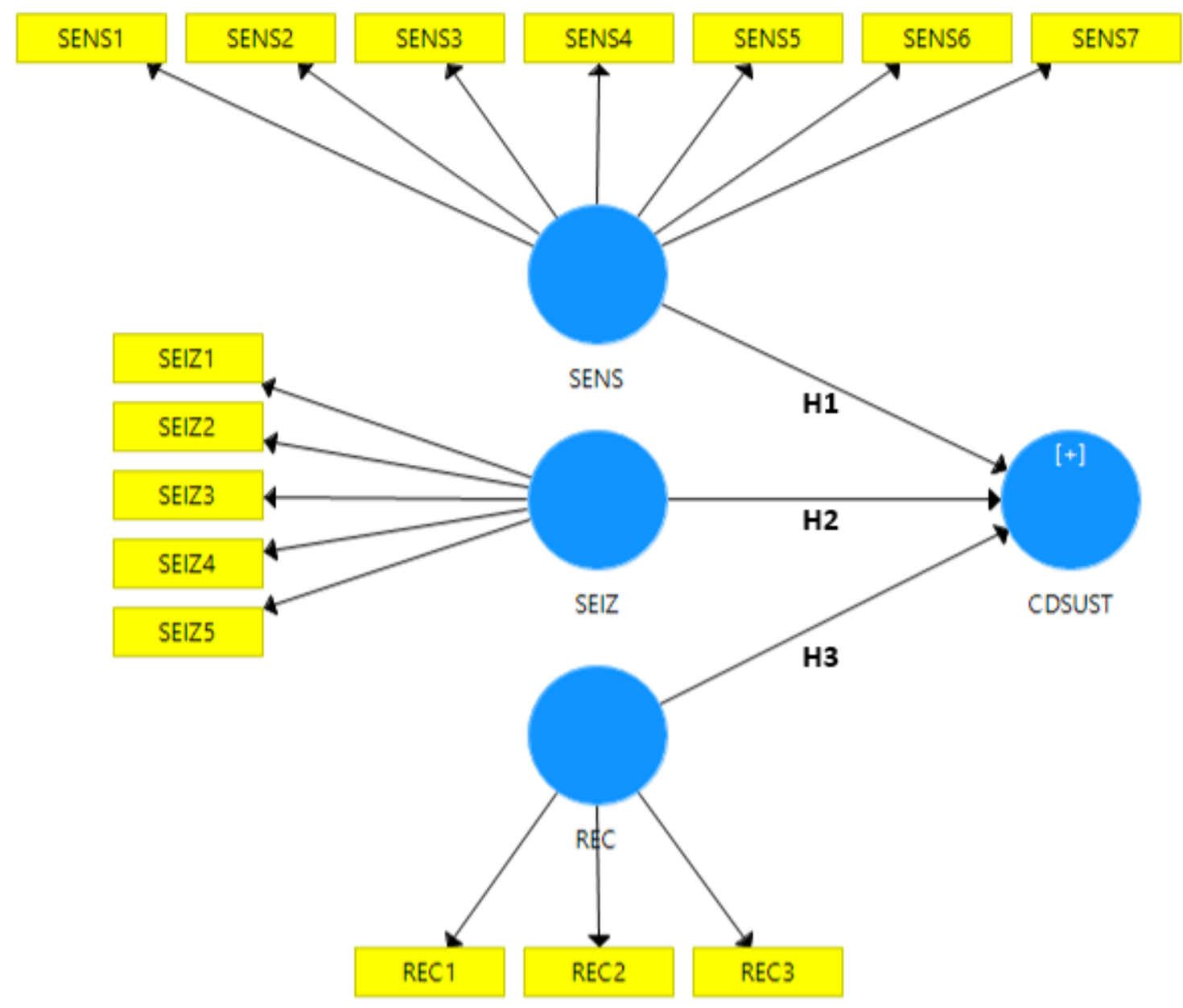

Nota 1: CDSUST é uma VL de segunda ordem.

Nota 2: A VL CDSUS contém 15 indicadores (os indicadores das 3 VLs de primeira ordem foram repetidos nela), mas foram escondidos (hide indicators) para facilitar a visualização do modelo.

$$
\text { Fonte: Elaboração própria. }
$$

\section{Modelo de Mensuração}

As análises do modelo de mensuração basearam-se nos pressupostos de Hair et al. (2014), quando os valores de referência são: Carga Fatorial $>0.4$, Variância Média Extraída $(\mathrm{AVE})>0.50$, Confiabilidade Composta $(\mathrm{CC})>0.70$ e Alpha de Cronbach $>0.70$. A Tabela 4 apre- 
A INFLUÊNCIA DAS CAPACIDADES DE DETECÇÃO, APREENSÃO E RECONFIGURAÇÃO NO DESENVOLVIMENTO DE CAPACIDADES DINÂMICAS ORIENTADAS À SUSTENTABILIDADE

Felipe Cavalheiro Zaluski - Clarice Vepo do Nascimento Welter - Jorge Oneide Sausen Clandia Maffini Gomes - Roberto Schoproni Bichueti

senta os valores encontrados para a validação convergente e indicadores de confiabilidade do modelo de mensuração.

Tabela 4 - Validação convergente e confiabilidade do modelo de mensuração

\begin{tabular}{|c|c|c|c|c|c|}
\hline VL 1a Ordem & Indicador & Carga Fatorial & AVE & CC & alpha \\
\hline \multirow{7}{*}{ SENS } & SENS1 & .827 & \multirow{7}{*}{.639} & \multirow{7}{*}{.925} & \multirow{7}{*}{.905} \\
\hline & SENS2 & .826 & & & \\
\hline & SENS3 & .707 & & & \\
\hline & SENS4 & .851 & & & \\
\hline & SENS5 & .814 & & & \\
\hline & SENS6 & .829 & & & \\
\hline & SENS7 & .729 & & & \\
\hline \multirow{5}{*}{ SEIZ } & SEIZ1 & .656 & \multirow{5}{*}{.601} & \multirow{5}{*}{.882} & \multirow{5}{*}{.831} \\
\hline & SEIZ2 & .898 & & & \\
\hline & SEIZ3 & .801 & & & \\
\hline & SEIZ4 & .715 & & & \\
\hline & SEIZ5 & .786 & & & \\
\hline \multirow{3}{*}{ REC } & REC1 & .893 & \multirow{3}{*}{.697} & \multirow{3}{*}{.873} & \multirow{3}{*}{.782} \\
\hline & REC2 & .787 & & & \\
\hline & REC3 & .821 & & & \\
\hline
\end{tabular}

Fonte: Elaboração própria.

Primeiramente analisou-se as cargas fatoriais e observou-se que todas as cargas fatoriais foram $>0.656$. Após, analisou-se a confiabilidade dos dados, verificando que todas os itens atingiram níveis satisfatórios > .873 para CC e > .782 para o Alpha de Cronbach. Além disso, as VLS de 1 a ordem atingiram a validade convergente porque suas medidas AVE > .500 (FORNELL; LARCKER, 1981).

Em seguimento, avalia-se a validade discriminante do modelo de mensuração. Segundo Hair et al. (2014), o modelo tem validade discriminante se cada um dos indicadores obtiver cargas superiores em suas próprias VLs do que em qualquer outra VL. Com os resultados das cargas cruzadas é possível verificar que todos os indicadores das VLs de 1a ordem têm valores superiores relativos aos correspondentes (cross loadings), o que assegura a sua validade discriminante.

Outro critério para a validade discriminante das VLs é analisar os valores na diagonal principal (VAVE), que devem ser superiores aos valores nas respectivas linhas e colunas (correlações), evidenciando a presença de validade discriminante (HAIR et al., 2014). Observa-se, na Tabela 5, que nenhuma correlação foi superior ao valor da raiz da AVE, confirmando a validade discriminante dos dados. 
A INFLUÊNCIA DAS CAPACIDADES DE DETECÇÃO, APREENSÃO E RECONFIGURAÇÃO NO DESENVOLVIMENTO

DE CAPACIDADES DINÂMICAS ORIENTADAS À SUSTENTABILIDADE

Felipe Cavalheiro Zaluski - Clarice Vepo do Nascimento Welter - Jorge Oneide Sausen Clandia Maffini Gomes - Roberto Schoproni Bichueti

Tabela 5 - Validação discriminante do modelo de mensuração

\begin{tabular}{lcccc}
\hline & VL 1a Ordem & 1 & 2 & 3 \\
\hline 1 SENS & .799 & & \\
2 SEIZ & .771 & .775 & .835 \\
3 REC & .794 & .732 & .832 \\
\hline
\end{tabular}

Legenda: matriz de correlações entre as VL com a raiz quadrada da AVE na diagonal em negrito. Todas as correlações são significantes a $1 \%$.

Fonte: Elaboração própria.

\section{Modelo Estrutural}

De acordo com Hair et al. (2014), para a análise do modelo estrutural e teste das hipóteses deve-se utilizar a técnica de Bootstrapping (5.000 reamostragens) e Blindfolding (distância de omissão de 8). Os resultados confirmam que o modelo estrutural tem relevância preditiva satisfatória, pois obteve valor de $Q^{2}>.000$ para todas as VLs e valores de $f^{2}$ com médios e grandes efeitos, demonstrando que o modelo tem acurácia e que os constructos são importantes para o ajuste geral do modelo. Os valores de $\mathrm{R}^{2}$, que explicam a variância das variáveis endógenas explicadas pelo modelo estrutural, têm um efeito grande (COHEN, 1997).

Os resultados das análises das hipóteses são apresentados na Tabela 6. Nota-se que as VLd de 1a ordem apresentam significância com a VL de 2a ordem, confirmando o modelo estrutural com significância estatística $(p<.05)$ e as hipóteses propostas.

Tabela 6 - Significância das relações estruturais

\begin{tabular}{cccccc}
\hline Hipóteses & Relações Estruturais & $\boldsymbol{\beta}$ & t-value & Sig. & Resultado \\
\hline H1 & SENS $\rightarrow$ CDSUST & .523 & 32.52 & .001 & Confirmada \\
H2 & SEIZ $\rightarrow$ CDSUST & .331 & 17.10 & .001 & Confirmada \\
H3 & REC $\rightarrow$ CDSUST & .223 & 16.91 & .001 & Confirmada \\
\hline
\end{tabular}

Fonte: Elaboração própria.

Observa-se que a Hipótese 1, que indica que a Capacidade de Detectar Oportunidades e Ameaças (sensing) influencia positivamente no desenvolvimento de Capacidades Dinâmicas Sustentáveis, foi confirmada ( $\beta=.523 ; p<.001$ ). A Hipótese 2 , que indica que a Capacidade de Apreensão de Oportunidades (seizing) influencia positivamente no desenvolvimento de Capacidades Dinâmicas Sustentáveis, foi confirmada ( $\beta=.331 ; p<.001$ ). Por fim, a Hipótese 3 , que indica que a Capacidade de Gerenciar Ameaças e Transformações (reconfiguring) influencia positivamente no desenvolvimento de Capacidades Dinâmicas Sustentáveis, foi confirmada $(\beta=.223$; $\mathrm{p}<.001)$.

\section{DISCUSSÕES}

Os resultados demonstram que as capacidades de detectar oportunidades e ameaças, apreensão de oportunidades e gerenciamento de ameaças e transformações, tem um efeito importante no desenvolvimento de CDs orientadas à sustentabilidade e podem facilitar a mudan- 
A INFLUÊNCIA DAS CAPACIDADES DE DETECÇÃO, APREENSÃO E RECONFIGURAÇÃO NO DESENVOLVIMENTO DE CAPACIDADES DINÂMICAS ORIENTADAS À SUSTENTABILIDADE

Felipe Cavalheiro Zaluski - Clarice Vepo do Nascimento Welter - Jorge Oneide Sausen Clandia Maffini Gomes - Roberto Schoproni Bichueti

ça estratégica das empresas em relação à sustentabilidade e maior vantagem competitiva em um ambiente de mercado em evolução (WU et al., 2012). Neste contexto, o desenvolvimento da sustentabilidade pode ser influenciado positivamente por uma série de capacidades que a empresa desenvolve ao longo de sua existência. Essas capacidades são fortemente relacionadas a fatores, como processos organizacionais, trajetória e posição da empresa (TEECE; PISANO; SHUEN, 1997; TEECE, 2007).

Percebe-se que o desenvolvimento da capacidade de detectar oportunidades e ameaças é relevante não apenas para reconhecer os possíveis riscos sustentáveis, como apontam Wu et al. (2012), mas, também, alinhar os aspectos ambientais e sociais com os objetivos econômicos da empresa. Estudos anteriores já argumentavam que, para alcançar a sustentabilidade, as empresas precisam detectar e reconhecer o potencial de oportunidades de sustentabilidade do mercado (HORBACH; OLTRA; BELIN, 2013; PORTER; VAN DER LINDE, 1995).

Para identificar as oportunidades orientadas para a sustentabilidade, é necessário que se desenvolvam vínculos de conhecimento com uma ampla gama de partes externas, pois empresas que, ativamente, reúnem informações de fontes externas, são mais propensas a inovar com o intuito de encontrar soluções com foco na sustentabilidade (MOUSAVI; BOSSINK; VAN VLIET, 2018). Assim, as capacidades de detectar oportunidades e ameaças têm o potencial de ajudar as empresas a superar informações incompletas sobre os desafios ambientais, com o objetivo de encontrar soluções sustentáveis.

Para, portanto, criar soluções sustentáveis, as empresas precisam de capacidades de deteç̧ão nos âmbitos tecnológico, do mercado e, também, ambiental. Elas devem olhar para as novas oportunidades tecnológicas com lentes para a sustentabilidade, e devem levar em conta o surgimento de normas e práticas ambientais, antecipando para manter sua posição de liderança (CASTIAUX, 2012).

O desenvolvimento da capacidade de apreensão de oportunidades sustentáveis demonstra a existência de procedimentos, estrutura e projetos que influenciam a definição de solução sustentável para os clientes e stakeholders. Ainda, delimita o modelo de negócio mais apropriado de forma a manter a oportunidade detectada em relação à sustentabilidade (NAGATA, 2020). Deste modo, para que as empresas desenvolvam capacidades dinâmicas orientadas à sustentabilidade, elas devem realizar atividades de apreensão, como formação de estratégias, atividades de introdução ao mercado, diálogos institucionais, atividades de coespecialização de recursos e redesenho de modelos de negócios (MOUSAVI; BOSSINK; VAN VLIET, 2017). Tal capacidade favorece a inovação em direção à sustentabilidade, pois aproveita fontes externas de conhecimento e informação (MOUSAVI; BOSSINK; VAN VLIET, 2018).

Por fim, para o desenvolvimento da capacidade de gerenciar ameaças e transformações sustentáveis, as organizações devem analisar o encerramento, modificação ou reconstrução de determinadas rotinas organizacionais e práticas que não estão alinhadas com a sustentabilidade (WU et al., 2012). O desenvolvimento de habilidades de transformação é considerado essencial para empresas inseridas em ambientes dinâmicos alcançarem desempenho superior em sustentabilidade (GRIGORESCU et al., 2019). Para que esse desenvolvimento ocorra é necessário que as empresas reconfigurem seus recursos que as ajudem a encontrar soluções fora de suas rotinas atuais, como novos métodos de organização de responsabilidades no trabalho, novas 
A INFLUÊNCIA DAS CAPACIDADES DE DETECÇÃO, APREENSÃO E RECONFIGURAÇÃO NO DESENVOLVIMENTO DE CAPACIDADES DINÂMICAS ORIENTADAS À SUSTENTABILIDADE

Felipe Cavalheiro Zaluski - Clarice Vepo do Nascimento Welter - Jorge Oneide Sausen Clandia Maffini Gomes - Roberto Schoproni Bichueti

práticas de negócios, novos métodos de organização de relações externas e orquestração do ecossistema de negócios (MOUSAVI; BOSSINK; VAN VLIET, 2017).

O entendimento de Wu et al. (2012) e lles e Martin (2013), em que as CDs orientadas à sustentabilidade se referem à capacidade das empresas de atender às expectativas sustentáveis em rápida evolução das partes interessadas, modificando intencionalmente os recursos funcionais para a busca simultânea de competências econômicas, ambientais e sociais, pode ser visualizado e corroborado. Elucidou-se que as empresas são mais capazes de trazer novas tecnologias e produtos para a sustentabilidade ao mercado de maneira eficaz quando desenvolvem e mobilizam suas capacidades dinâmicas em torno de questões de sustentabilidade.

As relações analisadas e confirmadas sugerem que a abordagem da CD é promissora para desenvolver um entendimento adicional do gerenciamento da sustentabilidade (AMUI et al., 2017; DARMANI; NIESTEN; HEKKERT, 2017; HOFMANN; THEYEL; WOOD, 2012; ILES; MARTIN, 2013). A influência das capacidades elencadas em Teece, Pisano e Shuen (1997) nos aspectos sustentáveis analisados, reforça a necessidade de que as organizações invistam no desenvolvimento de capacidades e recursos para o alinhamento de seus objetivos econômicos com orientação para a sustentabilidade (NAGATA, 2020). Desenvolver capacidades e recursos são necessários para que a empresa tenha atuação sustentável e vantagem competitiva de mercado.

\section{CONSIDERAÇÕES FINAIS}

Tomando 77 indústrias de grande porte da região metropolitana de Porto Alegre/RS como amostra de pesquisa e usando o modelo adaptado dos microfundamentos das CDs de Teece (2007) e os indicadores propostos por Nagata (2020), foi analisada a influência das capacidades de detectar oportunidades e ameaças, apreensão de oportunidades e gerenciamento de ameaças e transformações no desenvolvimento de Capacidades Dinâmicas orientadas à sustentabilidade.

Pode-se concluir que a articulação das capacidades propostas por Teece (2007), alinhadas com os conceitos de sustentabilidade, podem auxiliar as empresas no desenvolvimento de capacidades dinâmicas orientadas à sustentabilidade. Argumenta-se que se a empresa desenvolver a capacidade de detectar oportunidades e ameaças, apreensão de oportunidades e gerenciamento de ameaças e transformações, migrará para estratégias e operações mais sustentáveis que se traduzirão em viabilidade não somente econômica, mas, também, ambiental e social.

Desenvolver capacidades dinâmicas da empresa, portanto, é um caminho para a sustentabilidade em organizações. Estes achados estão alinhados com o estudo de Amui et al. (2017), ao afirmarem que promover a sustentabilidade como uma capacidade dinâmica organizacional é um desafio com o qual as empresas terão de lidar se quiserem manter-se competitivas. Corrobora, também, a afirmação de Shang et al. (2019), que inferem que, para melhorar o desempenho sustentável das empresas, é preciso foco no desenvolvimento de capacidades dinâmicas orientadas à sustentabilidade.

As conclusões deste estudo têm implicações teóricas e gerenciais. Com relação às implicações teóricas, o artigo contribui para o avanço dos estudos relacionados às capacidades dinâmicas e de sustentabilidade, demonstrando como as organizações, a partir da combinação 
A INFLUÊNCIA DAS CAPACIDADES DE DETECÇÃO, APREENSÃO E RECONFIGURAÇÃO NO DESENVOLVIMENTO DE CAPACIDADES DINÂMICAS ORIENTADAS À SUSTENTABILIDADE

Felipe Cavalheiro Zaluski - Clarice Vepo do Nascimento Welter - Jorge Oneide Sausen Clandia Maffini Gomes - Roberto Schoproni Bichueti

de seus recursos e rotinas, podem vir a fomentar o desenvolvimento de suas capacidades dinâmicas orientadas à sustentabilidade (AMUI et al., 2017; MOUSAVI; BOSSINK; VAN VLIET, 2018).

Quanto às contribuições gerenciais, entender como as empresas podem utilizar as capacidades de detectar oportunidades e ameaças, apreensão de oportunidades e gerenciamento de ameaças e transformações no desenvolvimento de Capacidades Dinâmicas orientadas à sustentabilidade, pode vir a auxiliar as empresas a elaborarem estratégias sustentáveis, ajudando-as no alcance de vantagem competitiva sustentável.

Por fim, este estudo apresenta algumas limitações. Assume-se como limitação o fato de o estudo não poder ser generalizado para indústrias de outras regiões, pois foi realizado no segmento industrial de grande porte da região metropolitana do Estado do Rio Grande do Sul, com realidades particulares e com base na percepção dos funcionários destas empresas que ocupam cargos de nível estratégico das organizações. Ainda, o estudo teve como foco de análise o ambiente interno, não contemplando demais stakeholders, como clientes, funcionários e fornecedores, que poderiam contribuir para enriquecer e ampliar os seus resultados.

Futuras pesquisas empíricas poderiam ser conduzidas para identificar os fatores influentes para execução eficaz das capacidades dinâmicas, bem como as fontes destas capacidades orientadas para a sustentabilidade corporativa (WU et al., 2012). Pesquisas futuras também podem ser realizadas para entender quais são as habilidades e conhecimentos que podem permitir que a sustentabilidade se torne uma competência estratégica para as organizações. Ainda, estudos que busquem identificar que tipo de capacidade dinâmica pode ser desenvolvida para superar, de maneira mais eficaz, os desafios emergentes da sustentabilidade, podem ser realizados (AMUI et al., 2017). Estes estudos podem ser relevantes, pois, conhecendo quais habilidades e conhecimentos são necessários, empresas dos diversos setores podem introduzi-los com o intuito de desenvolver capacidades dinâmicas orientadas à sustentabilidade que proporcionem que se tornem mais competitivas, atendendo os diversos desafios ambientais.

\section{REFERÊNCIAS}

ABRAMOVAY, R. Muito além da economia verde. São Paulo: Editora Abril. 2012.

ALBORT-MORANT, G.; LEAL-MILLÁN, A.; CEPEDA-CARRIÓN, G. The antecedents of green innovation performance: A model of learning and capabilities. Journal of Business Research, v. 69, n. 11, p. 4.912-4.917, 2016.

ALHADDI, H. Triple bottom line and sustainability: A literature review. Business and Management Studies, v. 1, n. 2, p. 6-10, 2015.

AMUI, L.; JABBOUR, C.; JABBOUR, A.; KANNAN, D. Sustainability as a dynamic organizational capability: a systematic review and a future agenda toward a sustainable transition. Journal of Cleaner Production, $v$. 142, n. 1, p. 308-322, 2017.

ANDREEVA, T.; CHAIKA, V. Dynamic Capabilities: what they need to be dynamic? St. Petersburg State University. São Petersburgo, 2006.

AVESANI, M. Sustainability, sustainable development, and business sustainability. In: Life Cycle Sustainability Assessment for Decision-Making. Methodologies and Case Studies, p. 21-38, 2020.

BARCELOS, E. J. B.V. O efeito do dinamismo ambiental na estratégia competitiva das empresas: um estudo de caso múltiplo. 2015. Dissertação (Mestrado em Administração) - Universidade Paulista - Unip, São Paulo, SP, Brasil, 2015.

BYGDAS, A. L. Enacting dynamic capabilities in distributed organizational environments. Proceedings of the EGOS Conference. Bergen, Norway, 2006.

CASTIAUX, A. Developing Dynamic Capabilities to Meet Sustainable Development Challenges. International Journal of Innovation Management, v. 16, n. 6, 2012. 
A INFLUÊNCIA DAS CAPACIDADES DE DETECÇÃO, APREENSÃO E RECONFIGURAÇÃO NO DESENVOLVIMENTO DE CAPACIDADES DINÂMICAS ORIENTADAS À SUSTENTABILIDADE

Felipe Cavalheiro Zaluski - Clarice Vepo do Nascimento Welter - Jorge Oneide Sausen Clandia Maffini Gomes - Roberto Schoproni Bichueti

CMMAD. Comissão Mundial Sobre Meio Ambiente e Desenvolvimento. Nosso futuro comum. 2. ed. Rio de Janeiro: Editora da Fundação Getúlio Vargas, 1991. (Tradução da 1a ed. de Our common future, 1988). COHEN, J. Statistical Power Analysis for the Behavioral Sciences. Revised Edition. New York: Academic Press, 1997.

COLLIS, D. J. Research note: how valuable are organizational capabilities? Strategic Management Journal, v. 15, Special Issue, p. 43-152, 1994.

CORDES-BERSZINN, P. Dynamic capabilities: How organisational structures affect knowledge processes. London, UK: Palgrave Macmillan, 2013.

DARMANI, A.; NIESTEN, E. M. M. I.; HEKKERT, M. P. Characteristics of investors in onshore wind power in Sweden. Environmental Innovation and Societal Transitions, v. 24, p. 67-82, 2017.

DOSI, G.; FAILLO, M.; MARENGO, L. Organizational Capabilities, Patterns of Knowledge Accumulation and Governance. Organization, v. 29, p. 8-9, p. 1.165-1.185, 2008.

EISENHARDT, K. M.; MARTIN, J. A. Dynamic Capabilities: What are they? Strategic Management Journal, v, 21, n. 10-11, p. 1.105-1.121, 2000.

FIERGS. Federação das Indústrias do Estado do Rio Grande do Sul. A sociedade gaúcha tem pressa. 2019. Disponível em: https://www.fiergs.org.br/noticia/sociedade-gaucha-tem-pressa. Acesso em: 15 mar. 2020.

FORNELL, C.; LARCKER, D. F. Evaluating Structural Equation Models with Unobservable Variables and Measurement Error. Journal of Marketing Research, v. 18, n. 1, p. 39-50, 1981.

FROEHLICH, C.; BITENCOURT, C. Proposição de um modelo teórico para capacidade de inovação sustentável. Revista Ciências Administrativas, v. 2, n. 2, p. 554-581, 2015.

GELHARD, C.; VON DELFT, S. The role of organizational capabilities in achieving superior sustainability performance. Journal of Business Research, v. 69, n. 10, p. 4.632-4.642, 2016.

GERADTS, T. H. J.; BOCKEN, N. M. P. Driving Sustainability-Oriented Innovation. Mit Sloan Management Review, v. 60, v. 2, p. 78-83, 2018.

GLOBOCNIK, D.; RAUTER, R.; BAUMGARTNER, R. J. Synergy or conflict? The relationships among organisational culture, sustainability-related innovation performance, and economic innovation performance. International Journal of Innovation Management, v. 24, n. 1, 2020.

GRIGORESCU, A.; MAER-MATEI, M. M.; MOCANU, C.; ZAMFIR, A.-M. Key Drivers and Skills Needed for Innovative Companies Focused on Sustainability. Sustainability, v. 12 n. 1, p. 1-14, 2019.

GUERRA, R. M. A.; TONDOLO, V. A. G.; CAMARGO, M. E. O que (ainda) podemos aprender sobre capacidades dinâmicas. Revista Ibero-Americana de Estratégia, v. 15, n. 1, p, 44-64, 2016.

HAIR, J. F.; HULT, G. T. M.; RINGLE, C. M.; SARSTEDT, M. A Primer on Partial Least Squares Structural Equation Modeling (PLS-SEM). Thousand Oaks: Sage, 2014.

HANSMANN, R.; MIEG, H. A.; FRISCHKNECHT, P. Principal sustainability components: empirical analysis of synergies between the three pillars of sustainability, International Journal of Sustainable Development \& World Ecology, v. 19, n. 5, p. 451-459, 2012.

HART, S. L. A natural-resource-based view of the firm. The Academy of Management Review, v. 20, n. 4, p. 986-1.015, 1995.

HART, S. L.; DOWELL G. A natural-resource-based view of the firm: fi fteen years after. Journal of Management, v. 37, n. 5, p. 1.464-1.479, 2011.

HELFAT, C.; FINKELSTREIN, S.; MITCHELL, W.; PETERAF, M.; SINGH, H.; TEECE, D.; WINTER, S. Dynamic Capabilities: Understanding Strategic Changes in Organizations. Malden: Blackwell Publishing, 2007.

HOFMANN, K. H.; THEYEL, G.; WOOD, C. H. Identifying firm capabilities as drivers of environmental management and sustainability practices - Evidence from small and mediumsized manufacturers. Business Strategy and the Environment, v. 21, n. 8, p. 530-545, 2012.

HORBACH, J.; OLTRA, V.; BELIN, J. Determinants and specificities of eco-innovations compared to other innovations - an econometric analysis for the French and German industry based on the community innovation survey. Industry and Innovation, v. 30, n. 3, p. 523-543, 2013.

HUBNER, G. P.; POWER, D. J. Retrospective reports of strategic managers: guidelines for increasing their accuracy. Strategic Management Journal, v. 6, p. 171-180, 1985.

IBGE. Instituto Brasileiro de Geografia e Estatística. Pesquisa Industrial Anual (PIA). 2019. Disponível em: https://www.ibge.gov.br/estatisticas/economicas/industria/9042-pesquisa-industrial-anual.html?=\&t=0-que-e. Acesso em: 19 jul. 2020. 
A INFLUÊNCIA DAS CAPACIDADES DE DETECÇÃO, APREENSÃO E RECONFIGURAÇÃO NO DESENVOLVIMENTO DE CAPACIDADES DINÂMICAS ORIENTADAS À SUSTENTABILIDADE

Felipe Cavalheiro Zaluski - Clarice Vepo do Nascimento Welter - Jorge Oneide Sausen Clandia Maffini Gomes - Roberto Schoproni Bichueti

ILES, A.; MARTIN, A. N. Expanding bioplastics production: Sustainable business innovation in the chemical industry. Journal of Cleaner Production, v. 45, p. 38-49, 2013.

LEDERWASCH, A.; MUKHEIBIR, P. The Triple Bottom Line and Progress Toward Ecological Sustainable Development: Australia's Coal Mining Industry as a Case Study. Resources, v. 2, n. 1, p. 26-38, 2013.

LEE, J.; LEE. K.; RHO, S. An evolutionary perspective on strategic group emergence: a genetic algorithm-based model. Strategic Management Journal, v. 23, n. 7, p. 727-746, 2002.

LOREDO, E.; LOPEZ-MIELGO, N.; PINEIRO-VILLAVERDE, G.; GARCÍA-ÁLVAREZ, M. T. Utilities: Innovation and Sustainability. Sustainability, v. 11, n. 4, p. 1-13, 2019.

MCKELVIE, A.; DAVIDSON, P. From Resourse Base to Dynamic Capabilities: an investigation of New Firms. British Journal of Management, v. 20, n. 1, p. 63-80, 2009.

MEIRELLES, D. S.; CAMARGO, A. A. B. Capacidades dinâmicas: O que são e como identificá-las? Revista de Administração Contemporânea, v. 18 (Ed. Esp), p. 41-64, 2014.

MOUSAVI, S.; BOSSINK, B.; VAN VLIET, M. Dynamic capabilities and organizational routines for managing innovation towards sustainability. Journal of Cleaner Production, v. 203, p. 224-239, 2018.

MOUSAVI, S.; BOSSINK, B.; VAN VLIET, M. Firms' capabilities for sustainable innovation: The case of biofuel for Aviation. Journal of Cleaner Production, v. 167, p. 1.263-1.275, 2017.

NAGATA, V. M. N. O efeito das capacidades dinâmicas na sustentabilidade: uma investigação multissetorial na perspectiva da teoria da visão baseada em recursos. 2020. Tese (Doutorado em Administração) - Faculdade de Economia, Administração e Contabilidade, Universidade de São Paulo, São Paulo, 2020.

PORTER, M. E.; VAN DER LINDE, C. Toward a new conception of the environment competitiveness relationship. The Journal of Economic Perspectives, v. 9, n. 4, p. 97-118, 1995.

PORTER, M. E.; KRAMER, M. R. Strategy \& society: the link between competitive advantage and corporate social responsibility. Harvard Business Review, v. 84, n. 12, p. 78-163, 2006.

PROTOGEROU, A.; CALOGHIROU, Y.; LIOUKAS, S. Dynamic capabilities and their indirect impact on firm performance. Industrial and Corporate Change, v. 21, n. 3, p. 615-647, 2011.

RENZI, A.; HENZ, A. P.; RIPPEL, R. Desenvolvimento econômico: do crescimento à prosperidade. Revista Brasileira de Gestão e Desenvolvimento Regional, v. 15, n. 6, p. 65-77, 2019.

REVILLA, M. A.; SARIS, W. E.; KROSNICK, J. A. Choosing the number of categories in agree-disagree scales. Sociological Research \& Methods, v. 43, n. 1, p. 73-97, 2014.

RICHARDSON, R. J. Pesquisa social: métodos e técnicas. 4. ed. São Paulo: Atlas, 2017.

RINGLE, C. M.; SILVA, D.; BIDO, D. Modelagem de equações estruturais com utilização do SMARTPLS. Revista Brasileira de Marketing - ReMark, v. 13, n. 2, p. 56-73, 2014.

ROLDÁN, J. L.; SÁNCHEZ-FRANCO, M. J. Variance-based structural equation modeling: Guidelines for using partial least squares in information systems research. In: MORA, M.; GELMAN, O.; STEENKAMP, A.; RAISINGHANI, M. (eds.). Research methodologies, innovations and philosophies in software systems engineering and information systems. Hershey, PA: Information Science Referenc., 2012. p. 193-221.

RUSSO, M. V.; FOUTS, P. A. A resource-based perspective on corporate environmental performance and profi tability. Academy of Management Journal, v. 40, n. 3, p. 534-559, 1997.

SHANG, H.; CHEN, R.; LI, Z. Dynamic sustainability capabilities and corporate sustainability performance: The mediating effect of resource management capabilities. Sustainable Development, v. 18, n. 4, p. 595612, 2019.

SUNDER, M. V.; GANESH, L. S.; MARATHE, R. R. Dynamic capabilities: a morphological analysis framework and agenda for future research. European Business Review, v. 31, n. 1, p, 25-63, 2019.

TEECE, D. Dynamic capabilities \& strategic management. Oxford: Oxford University Press, 2009.

TEECE, D. Explicating dynamic capabilities: the nature and microfoundations of (sustainable) enterprise performance. Strategic Management Journal, v. 28, n. 13, p. 1.319-1.350, 2007.

TEECE, D. J.; PISANO, G.; SHUEN, A. Dynamic capabilities and strategic management. Strategic Management Journal, v. 8, n. 7, p. 509-533, 1997.

TONDOLO, V. A. G.; BITENCOURT, C. C. Understanding Dynamic Capabilities from Its Antecedents, Processes and Outcomes. BBR - Brazilian Business Review, v. 5, n. 11, p. 122-144, 2014.

WANG, C. L.; AHMED, P. K. Dynamic capabilities: A review and research. International Journal of Management Reviews, Oxford, v. 9, p. 31-51, 2007.

WINTER, S. G. Economic "Natural Selection" and the Theory of the Firm. Yale Economic Essays, v. 4, p. 225-272, 1964. 
A INFLUÊNCIA DAS CAPACIDADES DE DETECÇÃO, APREENSÃO E RECONFIGURAÇÃO NO DESENVOLVIMENTO DE CAPACIDADES DINÂMICAS ORIENTADAS À SUSTENTABILIDADE

Felipe Cavalheiro Zaluski - Clarice Vepo do Nascimento Welter - Jorge Oneide Sausen

Clandia Maffini Gomes - Roberto Schoproni Bichueti

WINTER, S. G. Understanding Dynamic Capabilities. Strategic Management Journal, v. 24 n. 10, p. 991995, 2003.

WU, Q.; HE, Q.; DUAN, Y.; O’REGAN, N. Implementing dynamic capabilities for corporate strategic change toward sustainability. Strat. Change, v. 21, n. 5-6, p. 231-247, 2012.

ZAHRA, R.; GEORGE, G. Absorptive Capacity: A Review, Reconceptualization and Extension. Academy of Management Review, [S. I.], v. 27, n. 2, 2002.

ZAHRA, S. A.; SAPIENZA, H. J.; DAVIDSSON, P. Entrepreneurship and dynamic capabilities: a review, model and research agenda. Journal of Management Studies, v. 43, n. 4, p. 917-955, 2006.

ZALUSKI, F. C.; SAUSEN, J. O.; FERREIRA, G. C. Proposição de um instrumento de mensuração do desenvolvimento de capacidades dinâmicas. Iberoamerican Journal of Strategic Management (IJSM), v. 19, n. 2, p. 105-124, Apr./June 2020.

ZOLLO, M.; WINTER, S. G. Deliberate Learning and the Evolution of Dynamic Capabilities. Organization Science. v. 13, n. 3, p. 339-351, 2002. 